\title{
Usefulness of a preoperative inflammatory marker as a predictor of asymptomatic acute rejection after lung transplantation: a Japanese single-institution study
}

\author{
Takashi Kanou ${ }^{1}$, Masato Minami ${ }^{1}$, Naoki Wada $^{2}$, Soichiro Funaki ${ }^{1}$, Naoko Ose ${ }^{1}$, Eriko Fukui ${ }^{1}$, \\ Yasushi Shintani ${ }^{1}$ \\ ${ }^{1}$ Department of General Thoracic Surgery, Osaka University, Osaka, Japan; ${ }^{2}$ Department of Diagnostic Pathology, Graduate School of Medicine, \\ Osaka City University, Osaka, Japan \\ Contributions: (I) Conception and design: T Kanou; (II) Administrative support: None; (III) Provision of study materials or patients: T Kanou; (IV) \\ Collection and assembly of data: M Minami, N Wada, S Funaki, N Ose, E Fukui; (V) Data analysis and interpretation: T Kanou, Y Shintani; (VI) \\ Manuscript writing: All authors; (VII) Final approval of manuscript: All authors. \\ Correspondence to: Takashi Kanou. Department of General Thoracic Surgery, Osaka University, Osaka 565-0871, Japan. \\ Email: kanoutakashi0999@yahoo.co.jp.
}

\begin{abstract}
Background: Surveillance bronchoscopy $(\mathrm{SB})$ is performed as routine follow-up after lung transplantation (LTx), primarily for the early detection of clinically asymptomatic acute rejection (AR). To identify appropriate candidates for SB over a long period, we explored risk factors of asymptomatic AR after LTx.

Method: This study is a single-center and retrospective cohort study. Forty-five patients underwent cadaveric LTx between 2000 and 2016 in our institution. All enrolled patients had at least three months of follow-up. SB is scheduled at 1, 2, 3, 6, and 12 months after LTx routinely and annually thereafter until 5 years after LTx. A histological assessment for AR was performed according to the International Society for Heart and Lung Transplantation (ISHLT) criteria. The analysis of potential risk factors for AR was performed using a chi-square test and logistic regression analysis.

Results: The median period of follow-up after LTx for the entire cohort was 64 months. Asymptomatic AR (grade A1-A3) was detected in 22 patients, 14 of whom showed severe AR (worse than grade A2). The percentage of patients with AR was $5-24 \%$ at each time point, and $15 \%$ of patients still showed severe AR (A2 and A3) at 24 months after LTx. Potential risk factors included recipient factors (diagnosis, age, gender, BMI), donor factors (age, gender, smoking history, cause of brain death), HLA mismatch, operation-related factors, neutrophil-to-leucocyte ratio (NLR), platelet-to-leucocyte ratio (PLR), and other scores. Patients with a higher NLR showed a higher incidence of AR after LTx than others during follow-up ( $\mathrm{P}=0.01)$.

Conclusions: An increased perioperative NLR was significantly associated with a higher odds ratio of AR during follow-up. Patients with a high NLR seem to be good candidates for long-term SB.
\end{abstract}

Keywords: Lung transplantation (LTx); acute rejection (AR); surveillance bronchoscopy (SB); pathology

Submitted Mar 20, 2020. Accepted for publication Jul 24, 2020.

doi: $10.21037 /$ jtd-20-1325

View this article at: http://dx.doi.org/10.21037/jtd-20-1325

\section{Introduction}

Lung transplantation (LTx) is an established surgical treatment for patients with end-stage respiratory disease. Chronic lung allograft dysfunction (CLAD) is a significant cause of morbidity and one of the major limitations to the long-term survival after LTx (1). Severe acute rejection (AR) and human leukocyte antigen (HLA) mismatch are reported to be potent risk factors of CLAD (2). Therefore, the timely and accurate diagnosis of AR is required in order to optimize immunosuppressive therapy and preserve the 
pulmonary function of transplanted lungs (3).

AR after LTx is an important morbidity and occasional cause of mortality $(4,5)$. A transbronchial lung biopsy (TBLB) remains the gold-standard method for the diagnosis of rejection in transplanted lungs. Surveillance bronchoscopy (SB) is performed in many transplant centers as part of the routine follow-up protocol after LTx with the aim of not only investigating the presence of infection or airway disease but also detecting clinically asymptomatic AR early.

However, one major issue associated with SB is safety concerns, including risks of bleeding, pneumothorax, and pneumonia after bronchoscopy $(6,7)$. Due to the potential risk associated with $\mathrm{SB}$, appropriate candidates for $\mathrm{SB}$ must be selected. However, despite its clinical importance, the risk factors for asymptomatic AR remains unclear (8).

In the present study, our clinical question was which patients are at increased risk of asymptomatic AR and thus would benefit the most from long-term SB. To answer this question, we explored risk factors of asymptomatic $\mathrm{AR}$ in the surveillance protocol after LTx. We present the following article in accordance with the STROBE reporting checklist (available at http://dx.doi.org/10.21037/jtd-20-1325).

\section{Methods}

\section{Study design}

This study is a single-center and retrospective cohort study. The clinical characteristics of donors and recipients who underwent LTx in Osaka University Hospital were systematically investigated. The relationship between the occurrence of AR during follow-up after LTx and these clinical factors was analyzed.

The study protocol was approved by the Ethics Review Board for Clinical Studies at Osaka University (control number 10026) and individual consent for this retrospective analysis was waived. The study was conducted in accordance with the Declaration of Helsinki (as revised in 2013).

\section{Patients}

All patients underwent cadaveric LTx between 1999 and 2016 in our institution. All enrolled patients had at least three months of follow-up. All recipients received a threedrug regimen of calcineurin inhibitor (cyclosporine or tacrolimus), prednisolone, and mycophenolate mofetil. The serum concentrations of cyclosporine or tacrolimus were monitored using the trough level. Induction therapy was generally not performed in our institution.

The percentage of patients with AR was calculated by the ratio of $A R$ patients to the patients who had $\mathrm{SB}$ at each time point. The cumulative incidence of AR was also defined as the ratio of patients with a history of AR during follow-up to the entire cohort of this study.

\section{Surveillance schedule and TBLB procedures}

SB is scheduled at 1, 2, 3, 6, and 12 months after LTx routinely and annually thereafter until 5 years after LTx. Additional bronchoscopy procedures were performed if clinically indicated, including in cases of an abnormal shadow on chest tomography (CT) or the deterioration of the pulmonary function.

Bronchoscopic procedures were performed under general anesthesia with endotracheal intubation under spontaneous breathing. The basic technique was previously described (9). In brief, bronchoalveolar lavage (BAL) was routinely performed before a TBLB by injecting $30 \mathrm{~mL}$ saline three times into the right middle lobe or left lingular lobe. A lung biopsy was always performed under fluoroscopy. TBLB specimens were acquired from each segment in both the upper and lower lobes. At least 5 moderate size samples $(0.5-1 \mathrm{~mm})$ were taken during a single procedure in accordance with the guideline published in 2007 (10).

The histological assessment of all graft specimens with respect to $A R$ was performed by our pathologist (NW) according to the International Society for Heart and Lung Transplantation (ISHLT) criteria (10). When specimens diagnosed as grade $\geq \mathrm{A} 2$ were considered significant, we administered intravenous methylprednisolone $(15 \mathrm{mg} / \mathrm{kg} / \mathrm{d}$ for 3 days) or increased immunosuppressive agents.

\section{Statistical analyses}

Statistical analyses were performed using the JMP software program for Windows, version 14 (SAS Institute, Cary, NC, USA). The clinical factors of the donor and recipient were assessed as potential risk factors for the occurrence of AR during the entire follow-up period using the chi-square test as a univariate analysis. For the multivariate analysis, factors with a p-score $<0.1$ in the univariate analysis were included in a logistic regression analysis. Continuous variables were expressed as the median (range), and categorical variables were expressed as numbers. Regarding the preoperative blood test results, we divided the patients into two groups of higher and lower scores based on the median score. A p-score $\leq 0.05$ 
Table 1 Recipient and donor characteristics

\begin{tabular}{|c|c|}
\hline Variables & Numbers \\
\hline Total number & 45 \\
\hline \multicolumn{2}{|l|}{ Recipient gender, n (\%) } \\
\hline Male & $17[37]$ \\
\hline Recipient age (median) & 44 \\
\hline \multicolumn{2}{|l|}{ Diagnosis, n (\%) } \\
\hline LAM & 14 [31] \\
\hline IPF & $11[24]$ \\
\hline $\mathrm{PAH}$ & $10[22]$ \\
\hline Emphysema & 4 [9] \\
\hline Others & $6[14]$ \\
\hline Recipient BMI (median) & 18.4 \\
\hline Donor age (median) & 45 \\
\hline \multicolumn{2}{|l|}{ Cause of brain death, $n(\%)$} \\
\hline CVD / others & $26[57] / 19[43]$ \\
\hline Smoking history of donor, $\mathrm{n}(\%)$ & $18[40]$ \\
\hline HLA mismatch number (4-6), n (\%) & $30[66]$ \\
\hline \multicolumn{2}{|l|}{ Procedure, n (\%) } \\
\hline Single & 28 [62] \\
\hline Waiting time (days, median) & 956 \\
\hline LTX period (later than 2010), n (\%) & $31[69]$ \\
\hline Intraoperative CPB, n (\%) & 15 [33] \\
\hline Postoperative ECLS, n (\%) & $6[13]$ \\
\hline \multicolumn{2}{|l|}{ Preoperative blood test (median) } \\
\hline WBC & 7,100 \\
\hline Alb & 4.2 \\
\hline CRP & 0.12 \\
\hline NLR (Neu/Lym ratio) & 3.5 \\
\hline PLR (PLT/Lym ratio) & 128 \\
\hline PNI (Prognostic nutrition index) & 50 \\
\hline Follow-up period (months, median) & 64 [4-223] \\
\hline Numbers of TBLB (mean) & $6.5[0-9]$ \\
\hline
\end{tabular}

LAM, lymphangioleiomyomatosis; IPF, idiopathic pulmonary fibrosis; PAH, pulmonary arterial hypertension; BMI, body mass index; CVD, cerebral vascular disease; HLA, human leukocyte antigen; CPB, cardiopulmonary bypass; ECLS, extracorporeal life support; WBC, white blood cell; CRP, c-reactive protein; NLR, neutrophil-to-lymphocyte ratio; PLR, platelet-tolymphocyte ratio; PNI, prognostic nutrition index. was considered statistically significant in all comparisons.

\section{Results \\ Characteristics of the study population}

From January 1999 to December 2016, we performed 48 cases LTx procedures with cadaveric donation. We excluded three cases due to their short post-transplant survival time of less than 3 months. The clinical characteristics of the donor and recipient are shown in Table 1. In total, SB was performed in 294 members of our cohort. The median length of follow-up for each recipient was 64 months (range, 4-223 months), and the mean number of SB procedures during each follow-up period was 6.5. Twenty-eight patients received single LTx, and the median waiting time was 956 days.

\section{Percentage of patients with AR after LTx}

The results of SB according to the follow-up period after LTx are shown in Figure 1. The percentage of patients with AR was $5-24 \%$ at each time point. AR scores of A23 , which usually required clinical intervention to prevent graft dysfunction, were frequently detected not only within one year after LTx but later as well. Interestingly, $15 \%$ of patients still showed severe AR (A2 and A3) at 2 years after LTx. In addition, SB detected asymptomatic AR until five years after LTx.

\section{Predictors of asymptomatic AR after LTx}

We investigated the relationship between the incidence of $\mathrm{AR}$ and the clinical characteristics, including donor factors, recipient factors, and operation-related factors (Table 2). In the univariate analysis, patients with a high neutrophil-to-lymphocyte ratio (NLR) and platelet-tolymphocyte ratio (PLR) showed a higher incidence of AR after LTx than others during the follow-up period. A primary diagnosis of pulmonary artery hypertension $(\mathrm{PAH})$ tended to be associated with a reduced incidence of AR, but not to a statistically significant degree. In the multivariate analysis, a higher NLR was an independent risk factor for asymptomatic AR after LTx (Table 3).

\section{Cumulative incidence of AR after LTx}

Based on the SB findings, the cumulative incidence of AR 


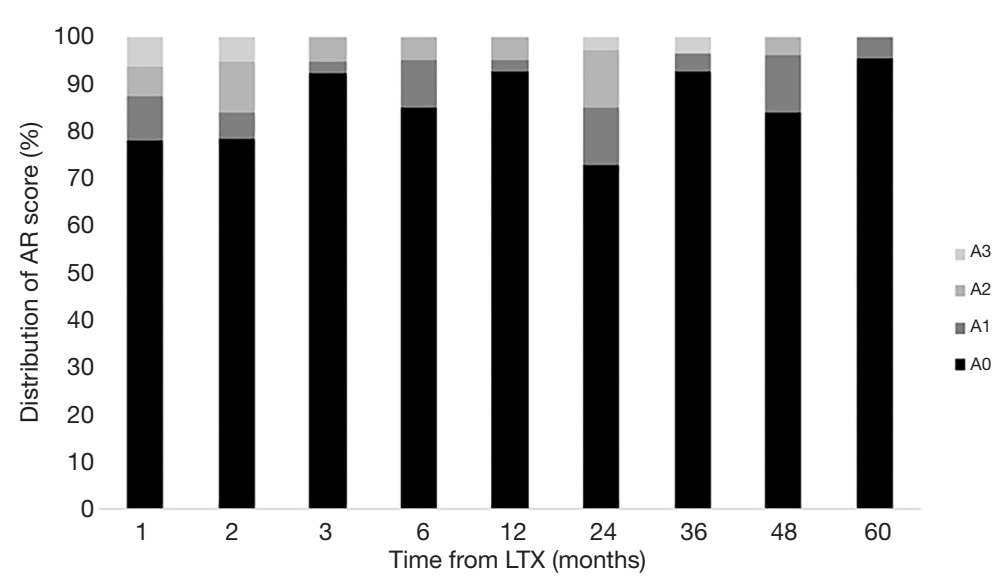

Figure 1 The results of SB according to the post-LTx period. Severe AR (A2-3) was frequently detected not only within 1 year after LTx but also beyond.

Table 2 Odds ratios of acute rejection (univariate analysis)

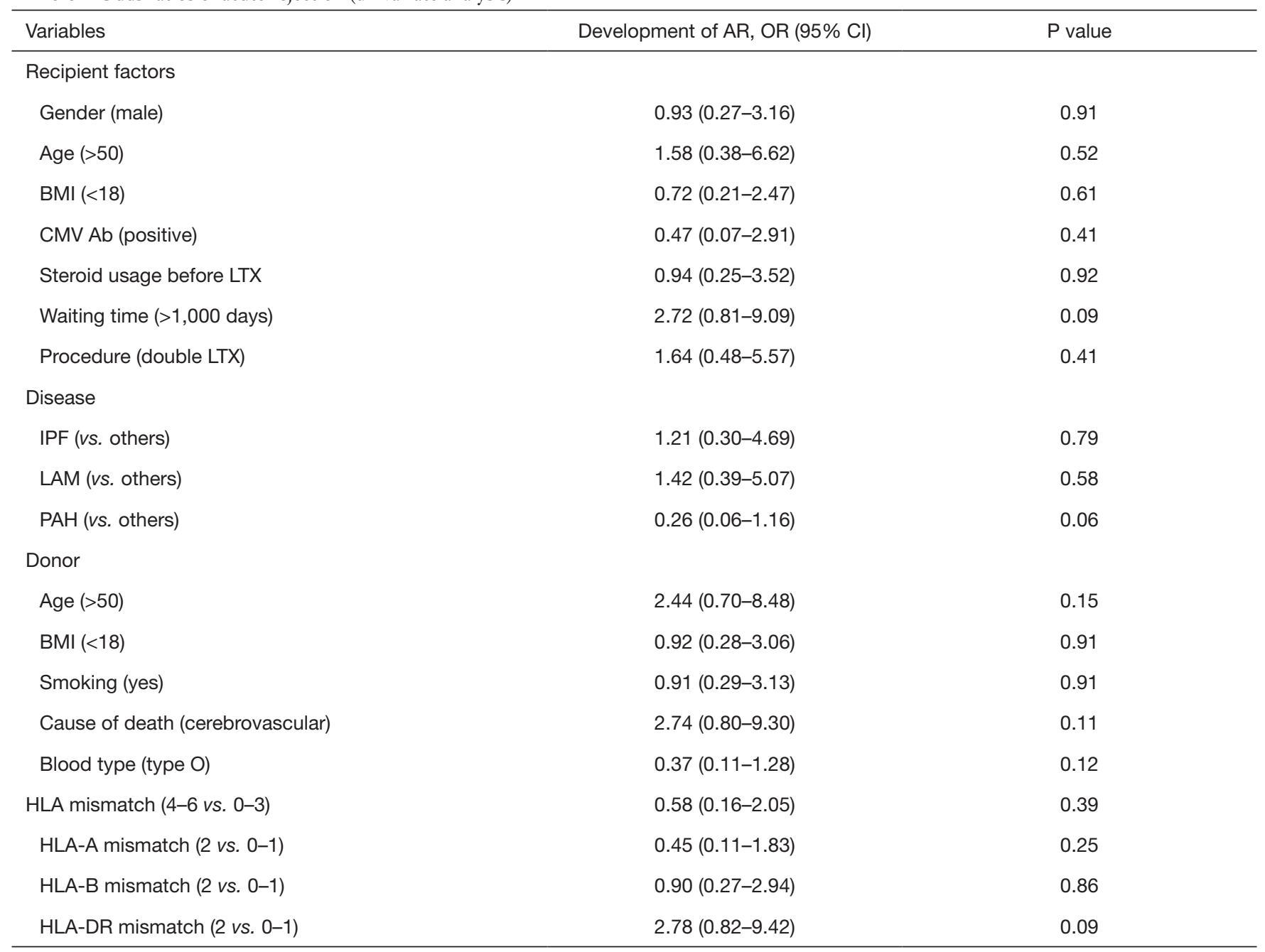

Table 2 (continued) 
Table 2 (continued)

\begin{tabular}{|c|c|c|}
\hline Variables & Development of AR, OR $(95 \% \mathrm{Cl})$ & $P$ value \\
\hline Period (2010 ) & $1.06(0.30-3.76)$ & 0.92 \\
\hline Ope time (>6 hours) & $1.30(0.40-4.20)$ & 0.66 \\
\hline CPB (intraoperative) & $0.50(0.14-1.79)$ & 0.29 \\
\hline Blood loss (>500 g) & $1.31(0.40-4.22)$ & 0.65 \\
\hline Re-thoracotomy & $0.32(0.07-1.45)$ & 0.12 \\
\hline Bronchial complication & $0.32(0.05-1.88)$ & 0.18 \\
\hline \multicolumn{3}{|l|}{ Preoperative blood test } \\
\hline $\mathrm{CRP}(>0.02)$ & $0.77(0.23-2.57)$ & 0.67 \\
\hline $\operatorname{NLR}(>3.5)$ & $4.95(1.38-17.71)$ & 0.01 \\
\hline $\operatorname{PLR}(>120)$ & $5.0(1.40-17.83)$ & 0.01 \\
\hline CAR $(>0.05)$ & $1.31(0.40-4.22)$ & 0.65 \\
\hline PNI $(<50)$ & $1.08(0.33-3.51)$ & 0.89 \\
\hline
\end{tabular}

BMI, body mass index; CMV, cytomegalovirus; IPF, idiopathic pulmonary fibrosis; LAM, lymphangioleiomyomatosis; PAH, pulmonary arterial hypertension; HLA, human leukocyte antigen; CPB, cardiopulmonary bypass; ECLS, extracorporeal life support; WBC, white blood cell; CRP, c-reactive protein; NLR, neutrophil-to-lymphocyte ratio; PLR, platelet-to-lymphocyte ratio; CAR, CRP-albumin ratio; PNI, prognostic nutrition index.

Table 3 Odds ratios of acute rejection (multivariate analysis)

\begin{tabular}{lcc}
\hline Variables & Development of AR, OR (95\% Cl) & P value \\
\hline Waiting time $(>1,000$ days) & $4.06(0.81-20.29)$ & 0.07 \\
PAH (vs. others) & $0.32(0.04-2.36)$ & 0.24 \\
HLA-DR mismatch (2 vs. 0-1) & $3.08(0.68-13.88)$ & 0.13 \\
NLR $(>3.5)$ & $5.48(0.97-30.86)$ & 0.04 \\
PLR $(>120)$ & $2.67(0.44-15.95)$ & 0.28 \\
\hline
\end{tabular}

$\mathrm{PAH}$, pulmonary arterial hypertension; HLA, human leukocyte antigen; NLR, neutrophil-to-lymphocyte ratio; PLR, platelet-to-lymphocyte ratio.

at each time point was calculated by the ratio of the patients who experienced AR during follow-up to all recipients. Our cohort included 23 patients (52\%) who experienced AR during the 5 -year follow-up period (Figure $2 A$ ). Interestingly, 2 patients were first found to have $A R$ at 24 months after LTx. In Figure 2B, the patients with higher NLR scores showed a higher cumulative incidence rate of
AR than those with lower NLR scores at 5 years' follow-up (65\% and $36 \%$, respectively).

\section{Discussion}

We found that higher scores of perioperative higher NLR was significantly associated with a higher odds ratio of 

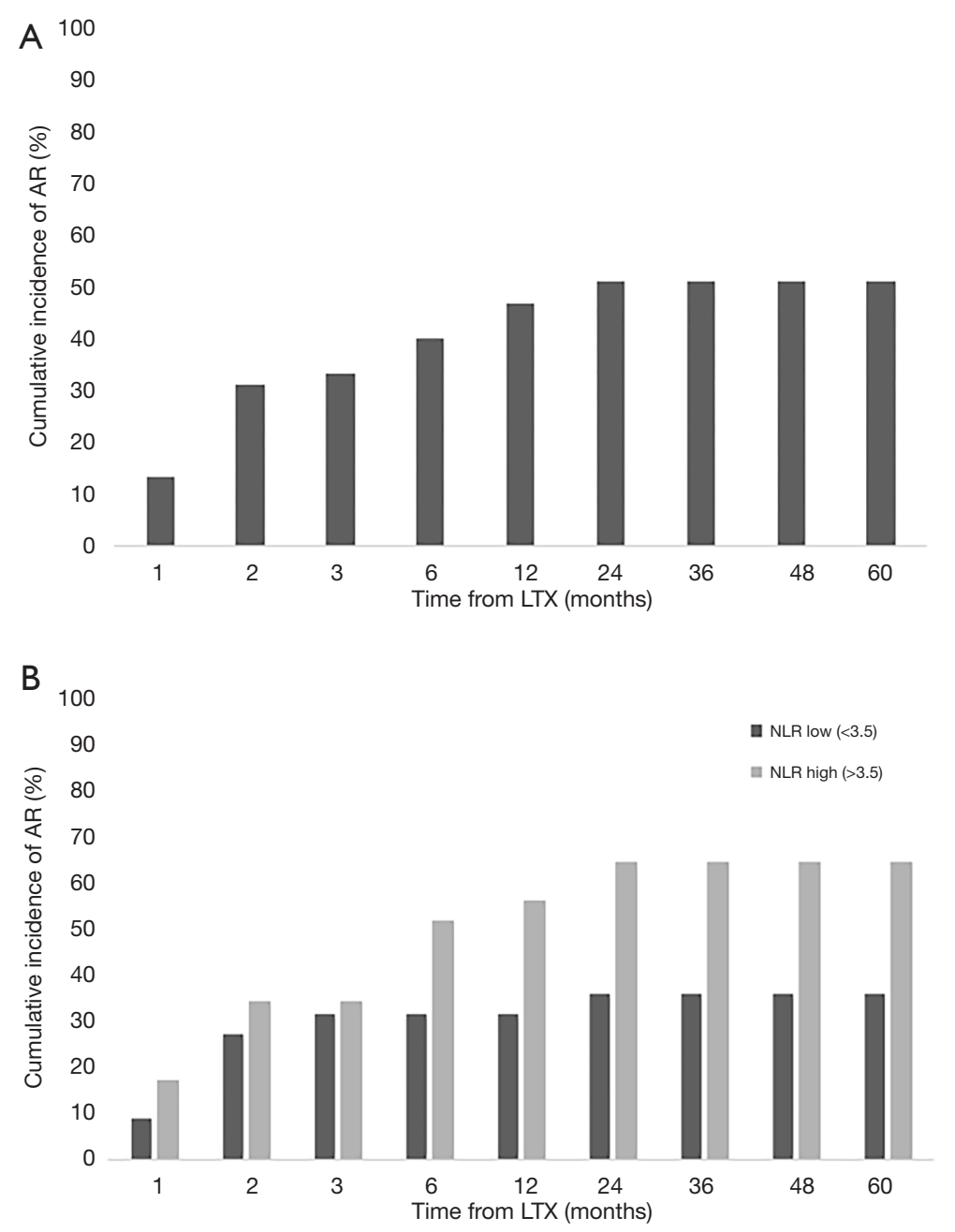

Figure 2 Cumulative incidence of AR. In our entire cohort, 23 patients (52\%) experienced AR during the 5-year follow-up period (A). The patients with higher NLR scores showed a higher cumulative incidence rate of AR in the 5-year follow-up period (B).

AR during the follow-up period. A high NLR might be a potential risk factor for the occurrence of AR after LTx. Our strategy including a long follow-up duration with periodic SB showed that AR could be detected not only within one year after LTx but later as well. Furthermore, to our knowledge, this is the first report to investigate appropriate candidates of long-term SB in Japan.

Several previous papers reported the ratio of patients found to have AR on SB within 1 year after LTx to be $20 \%$ to $30 \%(6,11)$. Similarly, our current study showed the ratio of $\mathrm{AR}$ at each time point to be around $15 \%$ to $25 \%$, and asymptomatic AR cases were detected even as long as 4 years after LTx. Furthermore, $12 \%$ of patients were found to have severe AR (more than grade 2) at 24 months after
LTx. In our institution, we perform lung biopsies from each lobe with more than 5 adequate samples, in accordance with the guideline (10). Based on those findings, we recommend SB be performed for at least two years after LTx in order to avoid missing asymptomatic AR.

The selection of appropriate candidates for $\mathrm{SB}$ is required because of the potential risk of complications with periodic SB (12). While many pretransplant donor and recipient characteristics have been studied, only a few have been found to be associated with an increased risk of AR (13). The recipient age, donor lung quality, and HLA matching are all considered potential pretransplant relative factors for AR. However, those factors did not show any significant difference in our analysis. 
We demonstrated in the present study the relationship between the preoperative recipient NLR, reflecting the immune function, and asymptomatic AR. Several previous reports have found that preoperative inflammatory markers, such as the NLR, were related to the outcome after solid organ transplantation $(14,15)$. Similarly, Kim et al. also reported that a high NLR score influenced the outcome after LTx in their survival analysis (16). Based on those previous findings, the recipient's preoperative inflammatory status is expected to be associated with the graft function and survival after organ transplantation. Recently, it was suggested that the inflammatory status might be related to the pathogenesis of graft dysfunction via inflammasome formation (17). However, no studies have investigated the relationship between the preoperative NLR and the occurrence of AR after LTx. The NLR is a simple, inexpensive, and readily available parameter in whole lung transplant candidates and may be a practical index for predicting patients at high risk of asymptomatic AR.

Several recent papers demonstrated the utility of novel biomarkers for diagnosing AR without a lung biopsy. The assessment of donor-derived cell-free DNA (ddcfDNA) in recipient plasma by single-nucleotide polymorphisms (SNPs) was suggested to be a promising biomarker for monitoring the rejection status after LTx $(18,19)$. A gene expression analysis for cell pellets of BAL was considered a meaningful diagnostic tool for AR after LTx (20). If those novel markers can be introduced for practical use, the diagnostic assessment of asymptomatic AR can be performed with much less invasiveness.

Several limitations associated with the present study warrant mention. First, our sample size is small, and this study is a single-institution, retrospective one. For a validation study, a different cohort collected separately and prospectively will be required to confirm our results. Second, it is difficult to elucidate the precise mechanisms underlying our findings despite several previous studies have shown that the preoperative NLR was related to the outcome after organ transplantation. Seropian et al. speculated that the innate inflammatory pathway was activated both before and after organ transplant and associated with the graft function (14). Further studies will be needed to uncover the mechanism underlying the relationship between AR and preoperative inflammatory markers, such as the NLR, which may lead to the discovery of novel therapeutic interventions against $A R$ and the prevention of graft dysfunction.

In conclusion, the current study showed that preoperative NLR was associated with the occurrence of AR after LTx. We expect our findings to be the first step in research to identify novel biomarkers of asymptomatic AR using universal parameters. Future studies should validate our results and confirm the findings. We expect this simple parameter to be beneficial for identifying patients at high risk of $\mathrm{AR}$ and to motivate us to perform $\mathrm{SB}$ for a long period after LTx.

\section{Acknowledgments}

We thank our colleagues from Osaka University Center of Medical Data Science and Advanced Clinical Epidemiology Investigator's Research Project for contributing their insight and expertise to our research.

Funding: None.

\section{Footnote}

Reporting Checklist: The authors have completed the STROBE reporting checklist. Available at http://dx.doi. org/10.21037/jtd-20-1325

Data Sharing Statement: Available at http://dx.doi. org/10.21037/jtd-20-1325

Conflicts of Interest: All authors have completed the ICMJE uniform disclosure form (available at http://dx.doi. org/10.21037/jtd-20-1325). The authors have no conflicts of interest to declare.

Ethical Statement: The authors are accountable for all aspects of the work in ensuring that questions related to the accuracy or integrity of any part of the work are appropriately investigated and resolved. The study protocol was approved by the Ethics Review Board for Clinical Studies at Osaka University (control number 10026) and individual consent for this retrospective analysis was waived. The study was conducted in accordance with the Declaration of Helsinki (as revised in 2013).

Open Access Statement: This is an Open Access article distributed in accordance with the Creative Commons Attribution-NonCommercial-NoDerivs 4.0 International License (CC BY-NC-ND 4.0), which permits the noncommercial replication and distribution of the article with the strict proviso that no changes or edits are made and the original work is properly cited (including links to both the 
formal publication through the relevant DOI and the license). See: https://creativecommons.org/licenses/by-nc-nd/4.0/.

\section{References}

1. Verleden GM, Vos R, Vanaudenaerde B, et al. Current views on chronic rejection after lung transplantation. Transpl Int 2015;28:1131-9.

2. Yamada $Y$, Langner T, Inci I, et al. Impact of human leukocyte antigen mismatch on lung transplant outcome. Interact Cardiovasc Thorac Surg 2018;26:859-64.

3. Benzimra M. Surveillance Bronchoscopy: Is It Still Relevant? Semin Respir Crit Care Med 2018;39:219-26.

4. Hodge G, Hodge S, Chambers D, et al. Acute lung transplant rejection is associated with localized increase in T-cell IFN $\gamma$ and TNF $\alpha$ proinflammatory cytokines in the airways. Transplantation 2007;84:1452-8.

5. Dabbs ADV, Hoffman LA, Iacono AT, et al. Are symptom reports useful for differentiating between acute rejection and pulmonary infection after lung transplantation? Heart Lung 2004;33:372-80.

6. McWilliams TJ, Williams TJ, Whitford HM, et al. Surveillance Bronchoscopy in Lung Transplant Recipients: Risk versus Benefit. J Heart Lung Transplant 2008;27:1203-9.

7. Hopkins PM, Aboyoun CL, Chhajed PN, et al. Prospective analysis of 1,235 transbronchial lung biopsies in lung transplant recipients. J Heart Lung Transplant 2002;21:1062-7.

8. Mangi AA, Mason DP, Nowicki ER, et al. Predictors of acute rejection after lung transplantation. Ann Thorac Surg 2011;91:1754-62.

9. Inoue $\mathrm{M}$, Minami $\mathrm{M}$, Wada $\mathrm{N}$, et al. Results of surveillance bronchoscopy after cadaveric lung transplantation: A Japanese single-institution study. Transplant Proc 2014;46:944-7.

10. Stewart S, Fishbein MC, Snell GI, et al. Revision of the 1996 Working Formulation for the Standardization of Nomenclature in the Diagnosis of Lung Rejection. J Heart Lung Transplant 2007;26:1229-42.

Cite this article as: Kanou T, Minami M, Wada N, Funaki S, Ose N, Fukui E, Shintani Y. Usefulness of a preoperative inflammatory marker as a predictor of asymptomatic acute rejection after lung transplantation: a Japanese single-institution study. J Thorac Dis 2020;12(9):47544761. doi: $10.21037 /$ jtd-20-1325
11. Chakinala MM, Ritter J, Gage BF, et al. Yield of surveillance bronchoscopy for acute rejection and lymphocytic bronchitis/bronchiolitis after lung transplantation. J Heart Lung Transplant 2004;23:1396-404.

12. Glanville A. The role of surveillance bronchoscopy post-lung transplantation. Semin Respir Crit Care Med 2013;34:414-20.

13. Koutsokera A, Levy L, Pal P, et al. Acute Cellular Rejection: Is It Still Relevant? Semin Respir Crit Care Med 2018;39:181-98.

14. Seropian IM, Romeo FJ, Pizarro R, et al. Neutrophilto-lymphocyte ratio and platelet-to-lymphocyte ratio as predictors of survival after heart transplantation. ESC Heart Fail 2018;5:149-56.

15. Kwon HM, Moon YJ, Jung KW, et al. Neutrophil-tolymphocyte ratio is a predictor of early graft dysfunction following living donor liver transplantation. Liver Int 2019;39:1545-56.

16. Kim CY, Kim SY, Song JH, et al. Usefulness of the preoperative prognostic nutritional index score as a predictor of the outcomes of lung transplantation: A single-institution experience. Clin Nutr 2019;38:2423-9.

17. Weigt SS, Palchevskiy V, Belperio JA. Inflammasomes and IL-1 biology in the pathogenesis of allograft dysfunction. J Clin Invest 2017;127:2022-9.

18. De Vlaminck I, Martin L, Kertesz M, et al. Noninvasive monitoring of infection and rejection after lung transplantation. Proc Natl Acad Sci U S A 2015;112:13336-41.

19. Tanaka S, Sugimoto S, Kurosaki T, et al. Donor-derived cell-free DNA is associated with acute rejection and decreased oxygenation in primary graft dysfunction after living donor-lobar lung transplantation. Sci Rep 2018;8:15366.

20. Weigt SS, Wang X, Palchevskiy V, et al. Usefulness of gene expression profiling of bronchoalveolar lavage cells in acute lung allograft rejection. J Heart Lung Transplant 2019;38:845-55. 